\title{
QD to NH; Mathematical Modelling of Nano-structure Formations
}

\author{
Jenö Takácsa* \\ Department of Engineering Science, University of Oxford.Parks Road, Oxford, OX1 3PJ, UK
}

Ákos Nemcsics

Institute for Microelectronics and Technology, Óbuda University, Tavaszmező u. 17. H-1084 Budapest, Hungary

\begin{abstract}
Mathematical formulation is given to the formations of the nano-structures created by DE (Droplet Epitaxy) for quantum - circuit application. Many of the known (and yet unknown) formations are derived from the two basic elements of nano-structures, namely the QD (Quantum Dot) and the NH (Nano Hole). Combined formations of the two shapes model the formations of the ones already used for circuit applications and predicts new nano-shapes for possible future usage such as meta materials. Their parameters are close related to the experimental conditions used at productions stage.
\end{abstract}

Keywords: Mathematical modelling; Modelling of nano-structure formations.

\section{(c) (9) CC BY: Creative Commons Attribution License 4.0}

\section{Introduction}

The role of the semiconductor based quantum-sized structures in the technology of quantum-circuit realization is on the increase. The importance of a novel technology called as DE (Droplet Epitaxy) is coming into focus for novel electronic devices and also quantum-circuit realization. DE technique allows the creations of not only single nano-structures but their complex combinations as well, which is a significant step in QD (Quatum Dot) circuit realization. Quantum computing is based on QD based technology, which fundamentally differs from any other earlier systems. This method is particularly useful when nano-structures should be produced in various shapes and sizes. The DE technology has advantages over other nano-structure preparation techniques and its flexible parameters make its introduction an important step, in number of applications. Like all technologies producing of nano-structures, DE is also a very complex physical process with interlinked physical parameters. The conditions, required by the production of nano-structures involve complex experimental details, with ca. ten inter-dependant parameters, such as substrate temperature, deposition rate, annealing time etc. For further details see [1]. The DE process mathematical formulation however demands an impossible task for mathematicians and researchers in physics alike. Some researchers, trying to use fuzzy logic, [2] leading to fuzzy inference system, based on geometrical quantum structure, [3] ended up with some dubious results. In case like that, natural scientists are often turning to a mathematical model, approximating the characteristic geometrical or physical attributes of the research subject in hand. Mathematical modelling has become an important and necessary tool in the arsenal of up to date natural science.

Here, we summarize the preliminaries and the technology of the DE based nanostructure preparation. The molecular beam epitaxy is the most advanced technology for nano-structure preparation. Till now, the only known method for the production of epitaxially grown QDs was the strain-induced method. In this way, two groups of shape formations, like pyramids and domes, can be created with defect-free QD transformations [4]. The DE is a viable alternative technology to the production of strain-driven QDs. In this technique, the material choice is not restricted by the lattice strain, which is a great advantage to a process, based on the strain-induced growth mode. DE also makes possible the fabrication of strain-free QDs and several other shaped nano-structures. This shape diversity of the produced nano-structures makes it advantageous in applications. The technology used for the growth governs the size, shape, and the elementary distribution of the developed structures. These physical parameters are very important in their applications. The technology of DE can be carried out by using of compound semiconductors with metallic and non-metallic components. In our case, we are using GaAs and related materials as sample medium. Here, the nano-structure preparation consists of two main parts such as the formation of metallic nano-sized droplet on the surface and its crystallization. In this way, not only conventional-shaped QDs but nano-hole (NH) and their combination can be created [1]. Geometrical data of these nano-sized structures is scanned by atomic force microscopy (AFM).

\section{First Fundamental Formation: QD}

One of the simplest fundamental shapes of nano-sized structure is the QD. The mode has two variable parameters to match up the AFM results for further mathematical analysis.

The numerical results produced by this model can be linked up with experimental parameters. The general formulation of the shape of the QD can be seen in the mathematical expression of (1). When $y_{Q D}$ represents the shape 
of the QD, then $a_{l}$ is the magnitude and $\square_{\square}$ is the shape factor and its shape and size can written in the following expression in normalized form as shown in (1):

$$
y_{Q D}=a_{1} \operatorname{sech}\left[\propto_{1} x\right]^{2}
$$

With $a_{l}=10$ and $\square \square \square \square \square \square \square(1)$ expression is plotted the 3D and 2D shape (cross section of 3D shape) of the QD as shown in Fig. 1.

The height of the QD is directly proportional to $a_{l}$. The $R$ radius of the base circle is inversely proportional to $\square \square \square \square \square$ see Fig. 5). $\square$ The proportionality factor is the function of the diminishing amplitude, where ever the user regards the end of the QD. This parameter is free choice for the user, because mathematically R is only zero at infinity.

The physical formation of this structure is based on Volmer-Weber clustering [1]. The AFM picture of such QDs are depicted in Fig. 2.

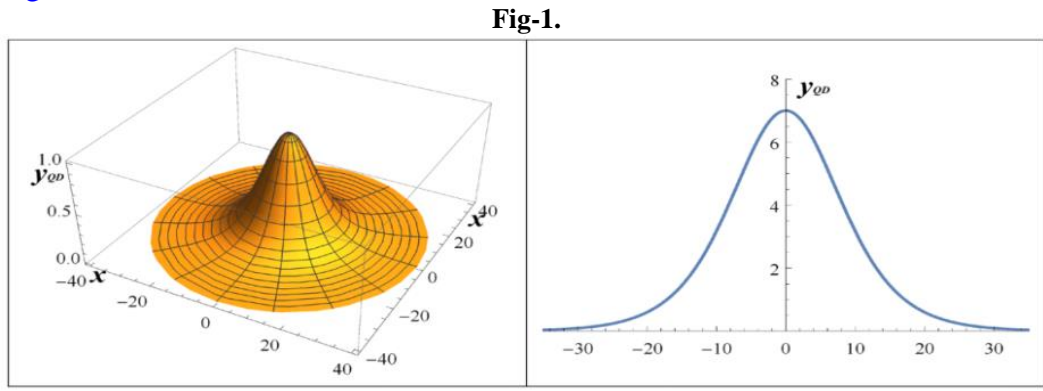

The 3D and the (cross-section) 2D images of a QD 3D and 2D are plots of (1).

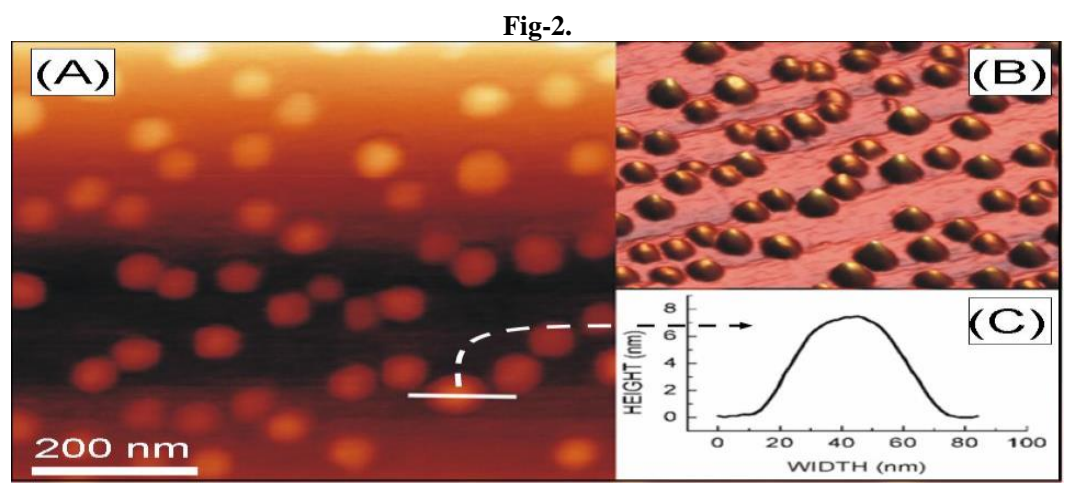

GaAs QD clusters grown on (001) oriented AlGaAs surface. During the DE process the typical parameters used are temperature $\left(200^{\circ} \mathrm{C}\right)$, Ga flux $(0.19 \mathrm{ML} / \mathrm{sec})$, waiting time $(60 \mathrm{sec})$, annealing $\left(350^{\circ} \mathrm{C}\right)$ at As pressure of $6 \times 10^{-5}$ Torr [5]. (A) top view and (B) tilted view of QD ensembles detected by AFM; (C) AFM line scans of QDs (marked with white line)

\section{Second Fundamental Formation: NH}

The model indication is that the second fundamental element of nano-structure is the NH (Nano - Hole), which combined with a QD, fundamentally governs the shape of the other large number of possible nano-object formations.

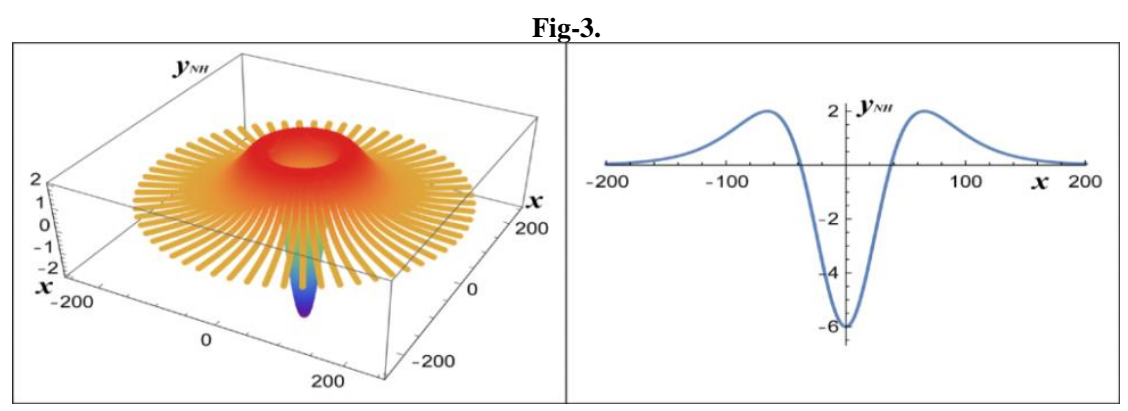

The 3D and the (cross-section) 2D images of a NH 3D and 2D are plots of (2) Fig. 4 depicts a group of NH-s with cylindrical lobe grown by DE process. Quantum-rings (QR) are formed by lobes around NHs. 3.75 ML Ga was deposited and annealing, carried out at $300^{\circ} \mathrm{C}$ under $4 \times 10^{-6}$ Torr arsenic pressure [6]. 


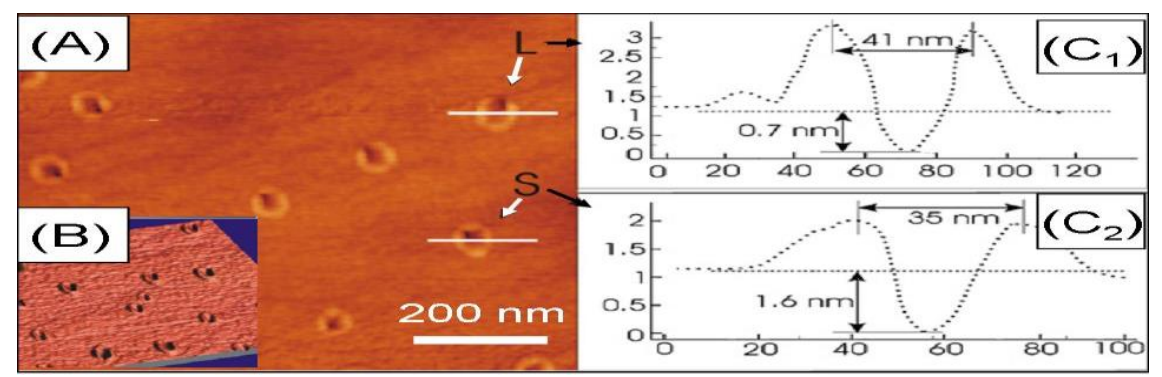

Group of NH-s [6]. (A) top view and (B) tilted view of NH ensembles detected by AFM. Left part of the figures $\left(\mathrm{C}_{1}\right.$ and $\left.\mathrm{C}_{2}\right)$, cross-section of the formations (line scan) marked with white lines

The mathematical expression modelling $\mathrm{NH}$ is described in (2).

$y_{N H}=-2 a_{2} \operatorname{sech}\left[\propto_{2} x\right]^{4}+4 a_{2} \operatorname{sech}\left[\propto_{2} x\right]^{2} \tanh \left[\propto_{2} x\right]^{2}$ 3 .

The model 3D and 2D image, resulting from (2), with $a_{2}$ and $\square \square_{\square} \square$ values as same as before, is depicted in Fig

The NH geometrical parameters like the radius of the base circle and the radius of the hole at $x=0$ and its peak, as well as the depth of the NH, can be calculated from (2). Their base the radius $R$, the radius of the QR and the radius of the hole at $\mathrm{x}=0$ dependence on $\square_{\square \square}$ is depicted in Fig.5. Physically, the structure formation based on the solution of the substrate material by liquid metallic component and also the surface diffusion of the components [1].

Fig-5.

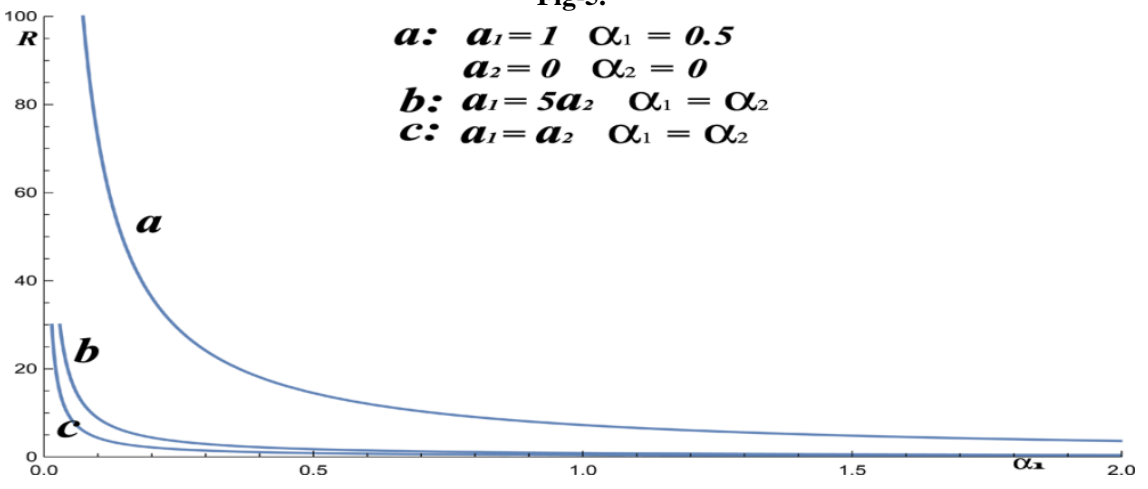

$R-s$ of Nano-Stuctures: a. Radius of QD base circle, b. Radius of QR ring, c. Radius of the hole made on the base by QR all as functions of $a_{l}$

\section{Combined QD-NH nano-structures}

The combination of the two basic formations is modelled by the following mathematical expression in (3).

$y_{A Q H}=a_{1} \operatorname{sech}\left[\propto_{1} x\right]^{2}-2 a_{2} \operatorname{sech}\left[\propto_{2} x\right]^{4}+4 a_{2} \operatorname{sech}\left[\propto_{2} x\right]^{2} \tanh \left[\propto_{2} x\right]^{2}$

\subsection{QR, Quantum Ring}

When in the combination the ratio between $a_{l}$, the QD amplitude and $a_{2}$, the amplitude of the NH, is $2: 1$ we can create a new $\mathrm{Q}$ formation, known as QR (Quantum Ring).

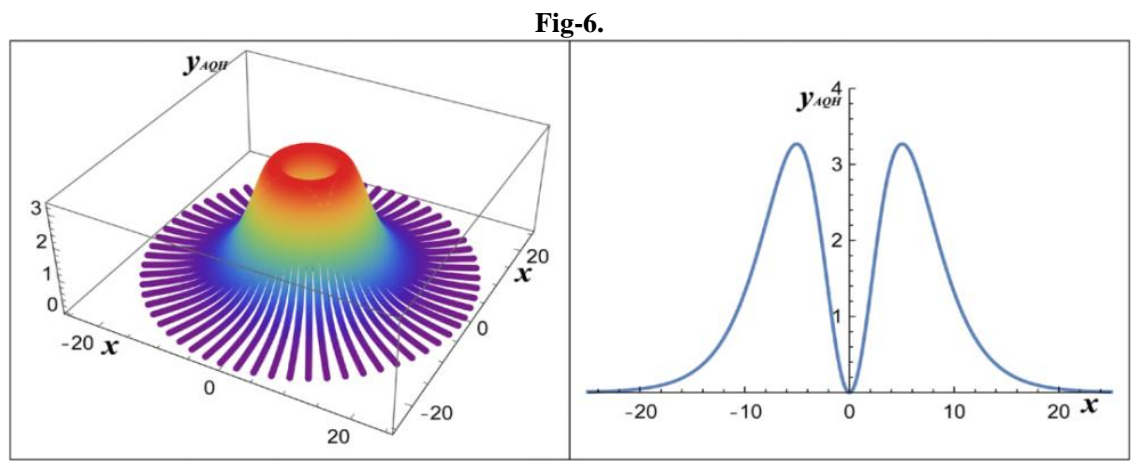

3D and the 2D cross section of a typical QR. 


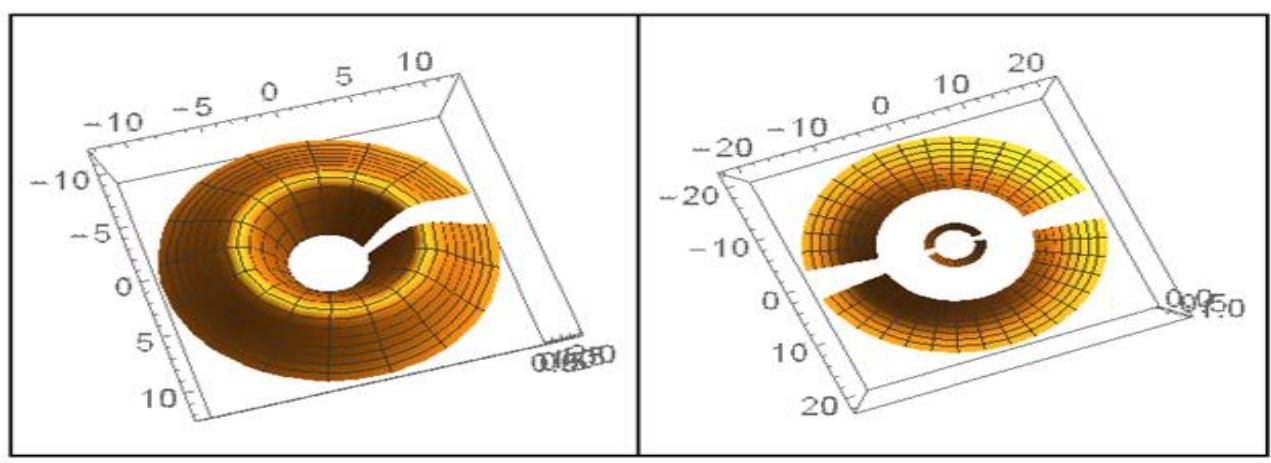

3D pictures of typical open and split double QR-s.

Fig.6 depicts the 3D and 2D picture of a type QR when the mathematical parameters are: $a_{1}=4.36, a_{2}=2.18$, $\square_{\square}=0.175, \square_{\square}=0.175$.

The mathematical formula modelling a typical $2 \mathrm{D} \mathrm{QR}$ is given in (4). $\tanh [0.175 x]^{2}$

$$
y_{A Q H}=4.36 \operatorname{sech}[0.175 x]^{2}-4.36 \operatorname{sech}[0.175 x]^{4}+8.72 \operatorname{sech}[0.175 x]^{2}
$$

When he QR is not closed it gets its use in the field of theory and application of meta-materials [7-9]. Fig.7 illustrates typical open QR and split QR-s, used to create meta-materials. Physical realization of a QR combined with $\mathrm{HN}$ is shown in Fig. 4.

\subsection{More Known Shapes}

During experimental processing two more Q based nano-structures were produced. One with an indentation on its top, representing a small presence of $\mathrm{NH}$ in the dominant Q, is pictured in Fig. 8. In this case $a_{2}$ is nearly an order of magnitude smaller than $a_{l}$.

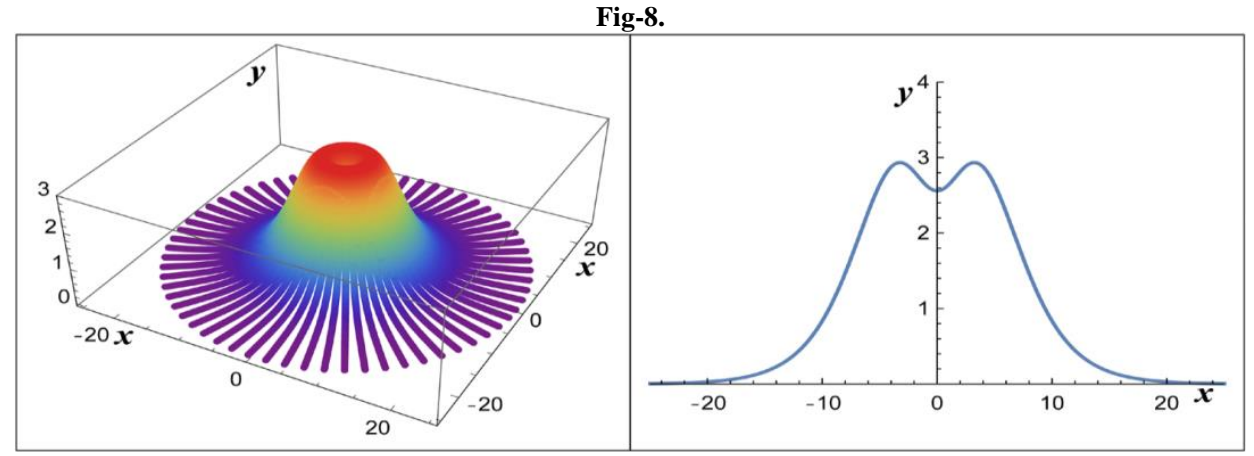

3D and 2D representation of a QD with small indentation.

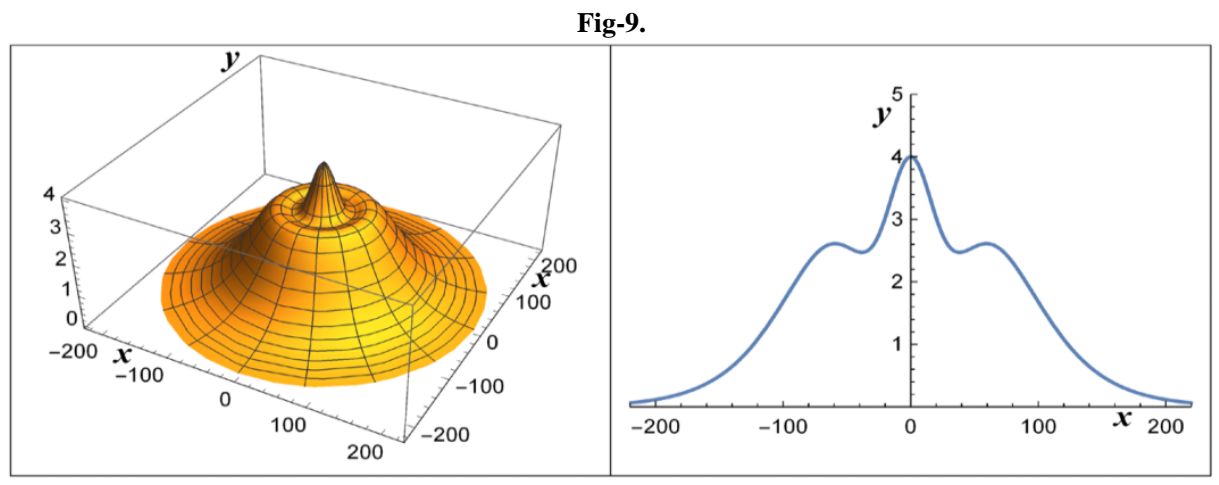

3D and 2D representation of a QD with shoulder.

For Fig. 8 the parameters are as follows: $a_{1}=4.5, a_{2}=1, \square_{\square}=0.175, \square_{\square}=0.175$ The other notable formation has a shoulder as shown in Fig.9. The model parameters for the formation above in Fig. 9 are as follows: $a_{1}=10, a_{2}=$ 3 ,

$=0.03, \square_{\square}=0.015$ Fig. 10. shows special formed nano-structures. Upper part of the figure shows AFM picture QD with shoulder, where $6 \mathrm{ML}$ was deposited at $350^{\circ} \mathrm{C}$. After the formation of Ga droplets, the sample was annealed in As atmosphere at $8 \times 10^{-7}$ Torr at $275^{\circ} \mathrm{C}$ for $60 \mathrm{~s}$, to form the ring and then at $5 \times 10^{-5}$ Torr at $150{ }^{\circ} \mathrm{C}$ for $5 \mathrm{~min}$ to form the dot [10-13]. While the lower part of the figure shows nano-ring with shoulder, where $1.5 \mathrm{ML}$ of $\mathrm{Ga}$ deposited at $400{ }^{\circ} \mathrm{C}$ and $\mathrm{Ga}-\mathrm{As}$ reaction carried out at $300{ }^{\circ} \mathrm{C}$ [13-15]

Fig-10. 


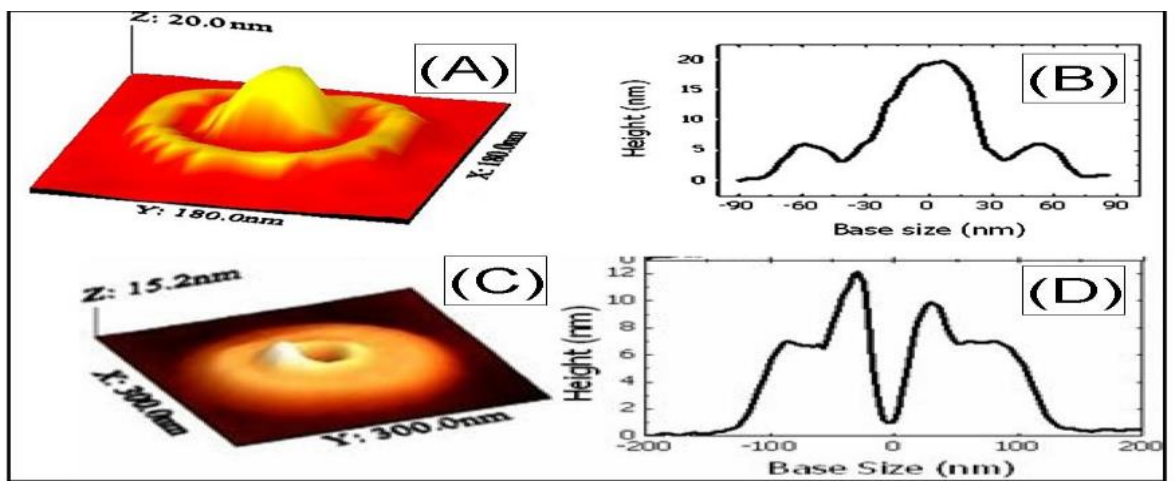

(A) AFM image of a QD with shoulder and (B) its AFM line scan

(C) AFM image of a nano-ring with shoulder and (D) its AFM line scan.

\section{Predicted Shapes}

With the combinations of 4 parameters $\left(a_{1}, a_{2}, \square_{\square} \square \square_{\square}\right.$, $)$ a number of known and used shapes can be modelled. Further to that the model predicts the existence of a number of so far unknown shapes. The previous examples indicate that with the appropriate physical parameters and experimental conditions, they can be produced. Here there are two samples of the formations, which spring into mind. This however does not represent a complete list of the possible Nano-structures, which predicted by the present model. These may represent a new line of research and possible new applications.

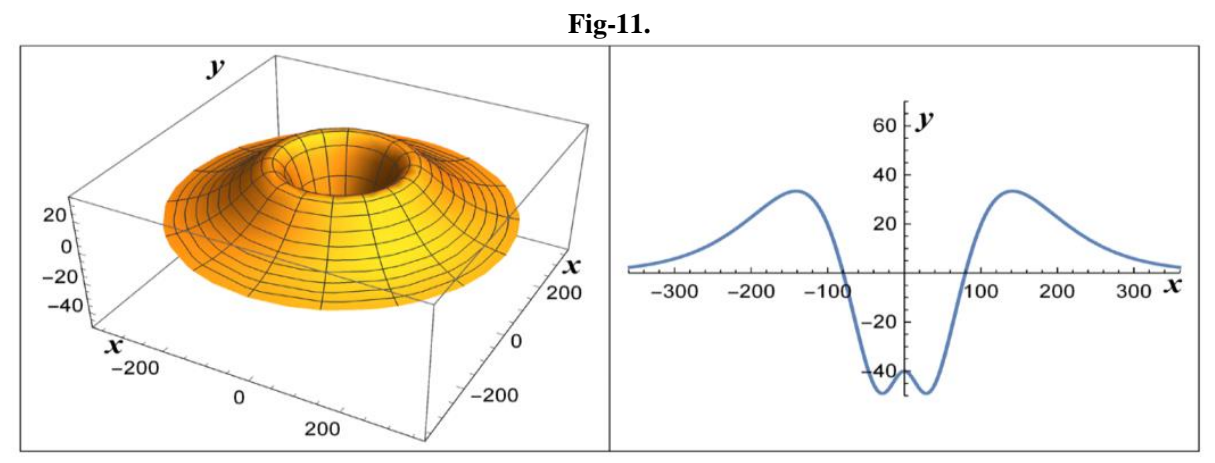

3D picture of a $\mathrm{Q}$ based nano formation and its $2 \mathrm{D}$ cross section, where $a_{Q D}$ is marginally exceeding $a_{N H}$ pictured as shape opposite of Fig 8.

The model parameters are: : $a_{1}=60, a_{2}=50, \square_{\square}=0.03, \square_{\square}=0.0081$

Fig-12.

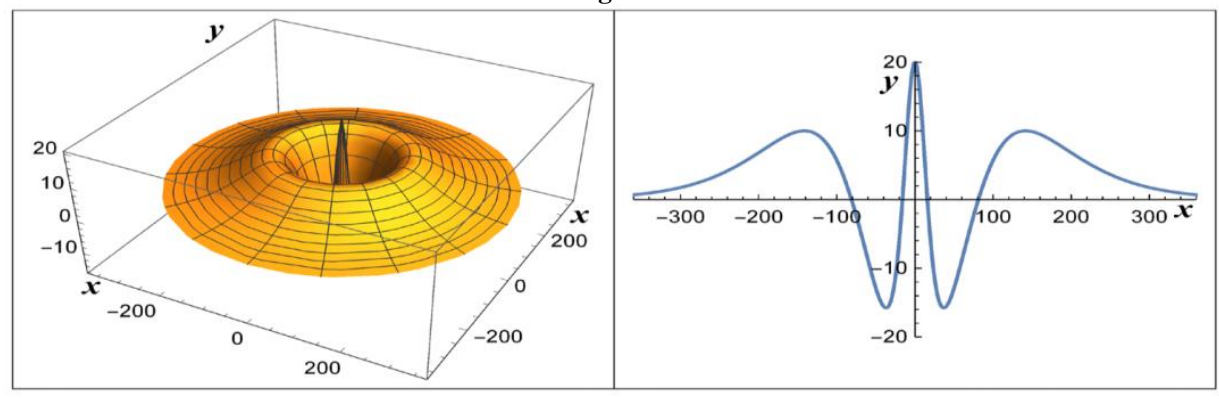

$3 \mathrm{D}$ and 2D picture of a predicted shape of a possible Nano-structure

The parameters for the formation in Fig. 12 are:: $a_{1}=50, a_{2}=15, \square_{\square}=0.05, \square_{\square}=0.0081$

\section{Conclusion}

A mathematical model of the shape of the Nano-structures can be a great help in choosing the right technological conditions and the physical parameters at planning. The energy - level created by the nano-object depends not only on the size and the material used, but also its shape. Once we can analytically model the object linked to its technological parameters we can gain on two ways. First it enables the user to calculate the energy levels associated with the nano-structure. Second, one can match the shape of nano - structure to the operating energy - levels to decide on the technological parameters for the required nano-structure.

The model proposed in the paper gives an insight into the possible $\mathrm{Q}$ based nano-shapes and predicts some unknown shapes, which may bring benefit into certain popular areas of future research and technology, like computers and meta-materials. 
Further experiments are planned to study the linkage between the experimental conditions and mathematical parameters of the model. The shapes predicted by the model may show the way to new applications [7-9, 16].

\section{References}

[1] Nemcsics, Á., 2015. Quantum dots prepared by droplet epitaxial method; chapter 5 in, quantum dots theory and applications. Rijeka, intech: N. Stavrou. pp. 119-149.

[2] Ürmös, A., Farkas, Z., Farkas, M., Sándor, T., Kóczy, L. T., and Nemcsics, Á., 2017. "Fuzzy and kohonen som based classification of different 0d nanostructures." In Conf. Proc. of IEEE 15th International Symposium on Applied Machine Intelligence and Informatics, Herlany, Slovakia (ed, A.Szakál) pp. 365369.

[3] Ürmös, A., Farkas, Z., Farkas, M., Sándor, T., T Kóczy, L., and Nemcsics, Á., 2017. "Application of selforganizing mapping for technological support of droplet epitaxy." Acta Polytechnica Hungarica, vol. 14, pp. 207-224.

[4] Lüth, H., 1998. Surfaces and interfaces of solid materials. Berlin: Springer Verlag.

[5] Nemcsics, Á., Tóth, L., Dobos, L., Heyn, C., Stemmann, A., Schramm, A., Welsch, H., and Hansen, W., 2010. "Composition of the "GaAs" quantum dot, grown by droplet epitaxy." Superlattices and Microstructures, vol. 48, pp. 351-357.

[6] Nemcsics, Á., Stemmann, A., and Takács, J., 2012. "To the understanding of the formation of the III-V based droplet epitxial nanorings." Microelectronics Reliability, vol. 52, pp. 430-433.

[7] Cutright, J. B., Joe, Y. S., and Hedin, E. R., 2012. "Transmission through multiple nanoscale rings with Zeeman-split quantum dots IEEE 15th international workshop on computational electronics " pp. 1-4.

[8] Plum, E., Liu, X.-X., Fedotov, V. A., Chen, Y., Tsai, D. P., and Zheludev, N. I., 2009. "Tuning chirality in photonic meta-materials with semiconductor quantum dots." In IEEE CLEO/Europe-EQEC 2009 European Conference on Lasers and Electro-Optics and the European Quantum Electronics Conference, Munich. pp. $1-1$.

[9] Zavvar, M. and Zavvari, M., 2017. "Design of split ring resonator for enhanced two-colour operation of quantum dot infrared photodetectors." IEEE Sensor Journal, vol. 17, pp. 4747-4551.

[10] Biccari, F., Bietti, S., Cavigli, L., Vinattieri, A., Nötzel, R., Gurioli, M., and Sanguinetti, S., 2016. "Temperature activated coupling in topologically distinct semiconductor nanostructures." J. Appl. Phys., vol. 120 , p.10.

[11] Sanguinetti, S., Somaschini, C., Bietti, S., and Koguchi, N., 2011. "Complex nanostructures by pulsed droplet epitaxy." Nanomater. Nanotechnol, vol. 1, pp. 14-17.

[12] Somaschini, C., Bietti, S., Koguchi, N., and Sanguinetti, S., 2011. "Coupled quantum dot-ring structures by droplet epitaxy." Nanotechnology, vol. 22, p. 5.

[13] Wu, J. and Wang, Z. M., 2014. "Droplet epitaxy for advanced optoelectronic materials and devices." $J$. Phys. D, Appl. Phys., vol. 47, p. 17.

[14] Lee, J. H., Wang, Z. M., Abuwaar, Z. Y., Strom, N. W., and Salamo, G. J., 2006. "Evolution between selfassembled single and double ring-like nanostructures." Nanotechnology, vol. 17, pp. 3973-3976.

[15] Somaschini, C., Bietti, S., Sanguinetti, S., Koguchi, N., and A., F., 2010. "Control of the lateral growth morphology in gaas droplet epitaxy." J. of Phys. Conference Series, vol. 245,

[16] Nemcsics, Á., 2018. Droplet epitaxy as a tool for the qd-based circuit realization, chapter 3 in, nonmagnetic and magnetic quantum dots. Rijeka, intech: N. Stavrou. pp. 43-59. 